Trägheitsmomente wurden schon zahlreiche theoretische Ansätze gemacht, die aber durchweg nicht voll befriedigen, da sie vielfach die Kopplungsglieder, deren zentrale Bedeutung hier aufgewiesen wurde, als vernachlässigbar betrachten und unterdrücken.

Im Sinne unserer Modellvorstellungen haben wir es hierbei mit Systemen von Massenpunkten mit meist recht großen Teilchenzahlen zu tun, bei deren Reduktion man überdies keineswegs mit dem Auftreten fester Zentralabstände rechnen kann. Es werden also Kopplungsglieder unvermeidlich auftreten. Es erhebt sich daher die Frage, wie man vorzugehen hat, um die Wirkung dieser Kopplungsglieder möglichst weitgehend zu beseitigen, um dann an einem möglichst gut ,entkoppelten“ System die auftretenden Energiestufen studieren zu können.

Unsere Überlegungen haben jedoch gezeigt, daß mit der hier angestrebten Entkopplung das Auftreten von Rotationsbanden im Energiespektrum des betreffenden Systems noch keineswegs verständlich wird. Hierzu sind vielmehr noch speziellere und noch näher zu untersuchende - Voraussetzungen erforderlich, die in unseren Überlegungen nur andeutungsweise sichtbar wurden. Ihre genauere Untersuchung an geeigneten Modellen soll einer weiteren Arbeit vorbehalten bleiben, die sich auch mit der Frage der ,effektiven“ Trägheitsmomente derartiger Gebilde beschäftigen soll.

\title{
Über Linear Passive Transformationen Stochastischer Prozesse I *
}

\author{
J. KELLER
}

Institut für Theoretische Physik der Rheinisch-Westfälischen Technischen Hochschule Aachen

(Z. Naturforsch. 23a, 1430-1438 [1968]; eingegangen am 13. Juli 1968)

\begin{abstract}
The theory of linear passive systems, developed by KönIG and Meixner, is extended to the case where the input is not a well determined function of time but rather a stochastic process. In this case the answer of the system generally will also be a stochastic process. The input and output processes are connected by a linear passive transformation (LPT). Some examples are given of physical systems which may be described by LPT of stochastic processes. General properties of the mean value and the dispersion of the output process are derived.
\end{abstract}

\section{Einleitung}

Viele Vorgänge in physikalischen Systemen lassen sich in mathematischer Hinsicht als deterministische oder stochastische Transformation $L(t)$ (das Argument $t$ beim Operator $L$ soll nur andeuten, daß dieser Operator auf Funktionen der Variablen $t$ wirkt) eines deterministischen oder stochastischen Prozesses $x(t)$ behandeln. Das heißt man kann den Vorgang schematisch durch eine Beziehung der Struktur

$$
y(t)=L(t) x(t)
$$

beschreiben. Dabei bezeichnet man die Größe $x(t)$ als ,,äußere Erregung“ oder Input-Funktion und $y(t)$ als ,Antwort des Systems" oder Output-Funktion. Der Operator $L(t)$ ist eine charakteristische Größe des vorliegenden physikalischen Systems. Je nachdem welche der in (E.1) auftretenden Größen

* Herrn Professor Dr. h.c. Dr. phil. J. Meixner zum 60. Geburtstag gewidmet.

1 Siehe z. B.: R. Kuвo, J. Phys. Soc. Japan 12, 570, 1203 [1957]. - S. NakaJima in ,Rendiconti della Scuola bekannt bzw. unbekannt sind, liegt eines der folgenden Probleme vor:

a) gegeben: $x(t), L(t)$; gesucht: $y(t)$,

b) gegeben: $x(t), y(t)$; gesucht: $L(t)$,

c) gegeben: $x(t)$ und einige allgemeine Eigenschaften von $L(t)$;

gesucht: Die explizite Gestalt von $L(t)$ und $y(t)$.

Bei Aufgabe a) liegt ein Vorhersageproblem vor: Wie wird ein bekanntes physikalisches System auf eine vorgegebene äußere Erregung reagieren? Beispiel: KuBo-Formalismus ${ }^{1}$.

Problemstellungen vom Typ b) liegen sehr oft in der Experimentalphysik vor: Man mißt die äußere Erregung und die Antwort des Systems und sucht daraus Aufschluß über das physikalische System selbst zu bekommen. Beispiel: Aufklärung der

Internationale die Fisica Enrico Fermi“", Corso X edited by S. R. DE Groot, Bologna 1959, p. 275 . - I. L. Lebowitz u. A. Shimony, Phys. Rev. 128, 1945 [1962]. F. V. Bunkin, Soviet Phys. JETP 17, 1954 [1963]. 
Struktur von Molekülen durch Kernresonanzexperimente ${ }^{2}$.

Bei Fragestellungen vom Typ c) liegt ein Parametrisierungsproblem vor. Man wird erwarten, daß die im Rahmen einer solchen Theorie möglichen Aussagen viel allgemeiner als die Aussagen bei Problemen vom Typ a) oder b) sein werden. Die Bedeutung dieser Art von Problemen für die Physik der Vorgänge scheint erst in jüngster Vergangenheit durch die Entwicklung der Theorie der Linear Passiven Systeme ${ }^{3,4}$ und deren Anwendung auf die Thermodynamik der Vorgänge ${ }^{5}$ entdeckt worden zu sein.

Unter den Transformationen $L(t)$ spielen die linearen eine besondere Rolle, weil sie einerseits zumindest einfacher als die nichtlinearen $\mathrm{zu}$ handhaben sind und andererseits viele Vorgänge eben durch lineare Gesetzmäßigkeiten beschrieben werden können. Unter den linearen Transformationen scheinen die passiven für die Physik der Vorgänge von besonderer Bedeutung zu sein. Der Begriff der deterministischen Linear Passiven Transformation (LPT) ist von KönIG und MeIXNeR ${ }^{3,4}$ entwickelt worden. Er wird in der vorliegenden Arbeit in etwas abgeänderter Form verwendet und in Kap. 1 erklärt werden. Unter einer Stochastischen Linear Passiven Transformation (SLPT) versteht man eine LPT, welche über einem Wahrscheinlichkeitsraum definiert ist. Dieser Begriff und seine Anwendung in der Physik der Vorgänge soll Gegenstand einer folgenden Arbeit sein.

Je nachdem, ob $x(t)$ und $L(t)$ deterministische oder stochastische - d.h. über Wahrscheinlichkeitsräumen definierte - Größen sind, erscheint es zweckmäßig, folgende 4 Fälle zu unterscheiden:

$L(t)$ determ. $L(t)$ stochastisch

\begin{tabular}{lll}
\hline$x(t)$ determ. & 1 & 2 \\
$x(t)$ stochastisch & 3 & 4 \\
\hline
\end{tabular}

2 A. Abragam, The Principles of Nuclear Magnetism, Clarendon-Press, Oxford 1961.

3 J. Meixner u. H. König, Rheol. Acta 2/3, 190 [1958]. H. König u. J. Meixner, Math. Nachr. 19, 265 [1958). H. KönIG, Arch. Math. 10, 447 [1959].

4 J. Meixner, Proc. IUPAP-Conf. on Statistical Mechanics and Thermodynamics, Aachen 1964, North Holland Publ. Co., Amsterdam 1965, p. 52.

5 J. Meixner, J. Appl. Mech. 33, 481 [1966].
Nur im Fall 1 ist die Größe $y(t)$ wieder deterministisch, in allen anderen Fällen wird $y(t)$ ein stochastischer Prozeß sein. Im folgenden seien zu jedem der Fälle 1-4 einige Beispiele von physikalischen Prozessen angeführt, welche sich durch LPT beschreiben lassen:

\section{Fall 1}

a) Der lineare gedämpfte harmonische Oszillator unter dem Einfluß einer äußeren Kraft $f(t)$ :

$$
m \ddot{x}+r \dot{x}+k x=f(t),
$$

$m$ Masse, $r$ Reibungskonstante, $k$ Federkonstante $x(t)$ Lagekoordinate des Oszillators.

b) Zahlreiche weitere Beispiele aus der Thermodynamik, Elektrodynamik und Quantenmechanik sind in ${ }^{4,5,6} \mathrm{zu}$ finden.

\section{Fall 2}

a) Alle unter 1 angeführten Prozesse, welche nun nicht mehr an Systemen mit determinierten, sondern mit statistisch verteilten Systemparametern ablaufen. Beispiel: Spannungs-Dehnungs-Beziehungen bei Materialproben mit statistisch verteilten elastischen Konstanten.

b) Der Kubo-Formalismus in der klassischen statistischen Mechanik bzw. in der Quantenmechanik ${ }^{1}$.

c) Kubo-MorI-Gleichung für statistische Mittelwerte?

d) Ausbreitung von Wellen in einem Medium mit räumlich statistisch veränderlichem Brechungsindex ${ }^{8}$.

\section{Fall 3}

a) Alle unter Fall 1 angeführten Prozesse, bei denen nunmehr $x(t)$ keine deterministische Funktion, sondern ein stochastischer Prozeß ist.

6 (a) J. Meixner, Arch. Rational Mech. Anal. 17, 278 [1964]. - (b) J. Meixner, J. Math. Phys. 4, 154 [1963]. - (c) J. MeIXner, Network Theory in its Relation to Thermodynamics, Proc. Symp. on Gerneralized Networks New York 1966, Vol. XVI in the Microwave Research Institute Symposia Series, Politechnic Press of the Polytechnic Institute of Brooklyn, N.Y.

7 H. Mori, Progr. Theor. Phys. 33, 423 [1965], 34, 399 [1965].

8 J. B. Keller, The Velocity and Attenuation of Waves in a Random Medium, Proc. 2nd Interdisciplinary Conf. on Electromagnetic Scattering, University of Massachusetts, Amherst 1965, Gordon and Breach 1967, p. 823 to 834 . 
b) Brownsche Bewegung eines Teilchens, beschrieben durch die (verallgemeinerte) Langevin-Gleichung

$$
\dot{v}(t)+\int_{-\infty}^{t} r(t-s) v(s) \mathrm{d} s=K(t),
$$

$K(t)$ statistische Kraft, welche den Einfluß der Stöße der Nachbarteilchen auf das Brownsche Teilchen beschreibt. $v(t)$ Geschwindigkeit des Teilchens. $r(s)$ Reibungsfunktion, welche folgende Eigenschaften haben soll:

1. $r(s)$ reell und positiv definit (vgl. ${ }^{14}, \mathrm{p} 65,74 \mathrm{ff}$.). Das heißt $r(s)$ ist eine in $0 \leqq s<\infty$ stetige und beschränkte Funktion für welche für alle Systeme von komplexen Konstanten $\left\{a_{i}\right\}$ und alle Systeme von reellen Konstanten $\left\{s_{i}\right\}$ mit $i=1 \cdots n$ gilt:

$$
\sum_{i, k=1}^{n} a_{i} * r\left(s_{i}-s_{\mathrm{k}}\right) a_{\mathrm{k}} \geqq 0 .
$$

2. $\int_{0}^{\infty} r(s) \mathrm{d} s<\infty$.

Unter diesen Voraussetzungen gilt $v(t)=L(t) \cdot K(t)$, wo $L(t)$ eine LPT ist.

c) Rauschen elektrischer Netzwerke (Widerstände, Elektronenröhren).

d) Materialien unter dem Einfluß einer statistischen äußeren Kraft. Zum Beispiel: Flugzeugflügel in turbulenter Atmosphäre ${ }^{9}$, Stoßdämpfer bei Fahrzeugen.

e) Bewegung elektrisch geladener Teilchen in statistischen elektromagnetischen Feldern ${ }^{10}$.

\section{Fall 4}

Materialproben mit statistisch verteilten Materialkonstanten unter dem Einfluß einer statistisch sich ändernden äußeren Kraft.

Wie erwähnt ist die Theorie der LPT zu Fall 1 von König und MeIXneR ${ }^{3,4}$ entwickelt und von ToBERGTE und HaCKENBROCH in 11, 12 fortgesetzt bzw. erweitert worden. Es ist das Ziel der vorliegenden Arbeit, diese Theorie auf den Fall 3 zu erweitern: Wir wollen in allgemeiner Weise (deterministische) LPT von stochastischen Prozessen untersuchen, da wir erwarten, daß diese Untersuchungen ein Hilfsmittel bei der Behandlung der unter Fall 3 auf-

9 V.S. Pugachev, Theory of Random Functions and its Application to Controll Problem, Pergamon Press, Oxford N.Y., 1. Auflage 1965, p. 413.

10 D. E. Hall u. P. A. Sturroch, Phys. Fluids 10, 6220 (1967). gezählten Prozesse sein werden. Zwei stochastische Prozesse, welche gemäß (E.1) miteinander durch eine LPT verknüpft sind, nennen wir ein Linear Passives Paar Stochastischer Prozesse (LPPSP). Nach genauer Definition der LPT und der LPPSP in Kap. 1 werden in Kap. 2 einige Aussagen über die Statistik des Prozesses $y(t)$ gemacht.

Auf einige Anwendungen der hier entwickelten Theorie zu Fall 3, speziell auf die Langevin-Gleichung (E.3), soll an anderer Stelle eingegangen werden.

\section{Definition Linear Passiver Transformationen und Linear Passiver Paare Stochastischer Prozesse}

Wie definieren zuerst den Begriff der Linear Passiven Transformation (LPT) und hernach den Begriff des Linear Passiven Paares Stochastischer Prozesse (LPPSP).

Sei $C^{(j)}$ der Raum aller komplexwertigen Funktionen $f(t)=f_{1}(t)+i f_{2}(t)$ mit folgenden Eigenschaften:

1) $f_{k}(t), k=1,2$ ist reell und besitzt $j$ viele stetige Ableitungen.

2) Für alle $n=0,1,2, \ldots, l=0,1 \ldots j$ und $k=1,2$ gilt

$$
\lim _{t \rightarrow-\infty} f_{k}^{(l)^{\prime}}(t) t^{n}=0 .
$$

Unter einer Linear Passiven Transformation $\mathfrak{\Omega}_{j}(t)$ einer Funktion $f(t) \in C^{(j)}$ versteht man eine Transformation, welche folgende Eigenschaften besitzt:

1. Bereichspostulat: Jeder Funktion $f(t) \in \mathrm{C}^{(j)}$ mit $j=0,1$ oder 2 wird eindeutig eine Funktion $g(t) \in \mathrm{C}^{(0)}$ zugeordnet. Man schreibt

$$
g(t)=\Omega_{j}(t) f(t) \quad \text { bzw. } \quad \mathfrak{Q}_{j}(t): f(t) \rightarrow g(t) .
$$

Im folgenden werde der Index $j$ einmal gewählt und dann festgehalten.

2. Linearität: Seien $f_{1}(t)$ und $f_{2}(t)$ zwei Funktionen aus $\mathrm{C}^{(j)}$ und seien

$$
g_{1}(t)=\Omega_{j}(t) f_{1}(t), \quad g_{2}(t)=\Omega_{j}(t) f_{2}(t)
$$

ihre Transformierten, so gilt für beliebige komplexe Konstante $\alpha_{1}$ und $\alpha_{2}$

$$
\Omega_{j}(t)\left[\alpha_{1} f_{1}(t)+\alpha_{2} f_{2}(t)\right]=\alpha_{1} g_{1}+\alpha_{2} g_{2} .
$$

11 H. König u. I. Tobergte, J. Reine Angew. Math. 212, $104,[1963]$.

12 W. Hackenbroch, Dissertation 1968, Universität des Saarlandes, Saarbrücken. 
3. Invarianz gegenüber Zeittranslation: Sei $f(t)$ eine Funktion aus $\mathrm{C}^{(j)}$ und sei $g(t)=\Omega_{j}(f) f(t)$ ihre Transformierte, so gilt für beliebige reelle $\tau$

$$
\Omega_{j}(t) f(t+\tau)=g(t+\tau) .
$$

4. Passivität: Sei $f(t)$ eine Funktion aus $\mathrm{C}^{(j)}$ und $g(t)$ ihre Transformierte, so gilt für alle $\tau$

$$
\operatorname{Re} \int_{-\infty}^{\tau} f^{*}(t) g(t) \mathrm{d} t \geqq 0
$$

Eine Kausalitätsbedingung - etwa $g(t)=0$ für $t \leqq t_{0}-$ wenn $f(t)=0$ für $t \leqq t_{0}$ braucht zu den Eigenschaften 1.-4. nicht mehr hinzugefügt werden, da sich eine solche aus 2 . und 4 . folgern läßt ${ }^{13}$.

Aus den Eigenschaften 1.-4. läßt $\operatorname{sich}^{3,4}$ folgende explizite Darstellung der Transformation $\mathfrak{L}_{j}(t)$ ableiten.

$$
\begin{aligned}
\Omega_{0}(t) f(t)= & {[\operatorname{Im} Y(1)-\dot{P}(+0)] f(t) } \\
& +\int_{0}^{\infty} \mathrm{d} s[b+P(s)-\ddot{P}(s)] f(t-s) \\
& \cdots f(t) \in \mathrm{C}(0) \\
\Omega_{1}(t) f(t)= & i \operatorname{Im} Y(1) f(t)+a \dot{f}(t) \\
& +\int_{0}^{\infty} \mathrm{d} s[b+P(s)] f(t-s) \\
& +\int_{0}^{\infty} \mathrm{d} s \dot{P}(s) \dot{f}(t-s) \cdots f(t) \in \mathrm{C}(1), \\
\Omega_{2}(t) f(t)= & i \operatorname{Im} Y(1) \dot{f}(t)+a \dot{f}(t) \\
& +\int_{0}^{\infty} \mathrm{d} s[b+P(s)] f(t-s) \\
& +\int_{0}^{\infty} \mathrm{d} s[P(0)-P(s)] \ddot{f}(t-s) \\
& \cdots f(t) \in \mathrm{C}(2) .
\end{aligned}
$$

Dabei bedeuten $Y(1)$ eine komplexe, $a$ und $b$ nichtnegative Konstante und $P(s)$ eine positiv definite ${ }^{14}$ Funktion, welche als charakteristische Funktion des Operators $\mathfrak{\Omega}_{j}(t)$ bezeichnet wird. Diese Funktion muß 2-j stetige Ableitungen besitzen. Bezüglich weiterer Eigenschaften der LPT sei auf Anhang A und ${ }^{3,4}$ sowie auf 6 a verwiesen.

Übt man nun eine LPT auf einen stochastischen Prozeß $x(t)$ aus - diese Operation wird im folgenden noch genau definiert - so erhält man einen neuen stochastischen Prozeß $y(t)$. Ein solches Paar von stochastischen Prozessen bezeichnen wir im

13 D. C. Youla, L. I. Castriota u. H. I. Carlin: IRE Trans. Circuit Theory 6, 102 [1959]. folgenden als Linear Passives Paar Stochastischer Prozesse (LPPSP). Ist der Operator $\mathfrak{\Omega}_{j}(t)$ einer LPT reell und ist der Prozeß $x(t)$ reell, so bezeichnet man die Prozesse $x(t)$ und $y(t)$ als reelles LPPSP.

Wir unterscheiden im folgenden zwischen der strengen linear passiven Transformation eines stochastischen Prozesses und der Transformation im statistischen Mittel.

a) Definition der strengen linear passiven Transformation eines stochastischen Prozesses.

Gegeben sei ein stochastischer Prozeß

$$
x(t)=\{x(\alpha, t)\},
$$

definiert über einem Wahrscheinlichkeitsraum

$$
\mathfrak{E}=\{\Omega(\zeta), F(\alpha, \beta \ldots), P(\alpha)\} .
$$

Dabei sei $\Omega(\zeta)$ die Menge der Elementarereignisse $\zeta$, $F(\alpha, \beta \ldots)$ ein Borelsches Ereignisfeld über den abgeleiteten Ereignissen $\alpha, \beta \ldots$ und $P(\alpha)$ ein Wahrscheinlichkeitsmaß für das Ereignis $\alpha$. Jede Realisierung $x(\alpha, t)$ des Prozesses $x(t)$ sei eine Funktion aus $\mathrm{C}^{(j)}$ mit $j=0,1$ oder 2 .

$$
x(\alpha, t) \in \mathrm{C}^{(j)} .
$$

Der Index $j$ werde wieder einmal gewählt und dann festgehalten. Ferner seien der statistische Mittelwert des Prozesses $x(t)$

$$
E_{x}(t)=\int_{\Omega(\zeta)} x(\alpha, t) \mathrm{d} P(\zeta)
$$

und alle Korrelationsfunktionen

$$
\begin{array}{r}
R_{x}^{(n)}\left(t, t_{1} \ldots t_{n-1}\right) \\
=\int_{\Omega(\zeta)} x(\alpha, t) x^{*}\left(\alpha, t_{1}\right) \ldots x\left(\alpha, t_{n-1}\right) \mathrm{d} P(\zeta)
\end{array}
$$

hinsichtlich jeder der Variablen $t, t_{1} \ldots t_{n-1}$ Funktionen aus $\mathrm{C}^{(j)}$.

$$
E_{x}(t), \quad R_{x}^{(n)}\left(t, t_{1} \ldots t_{n-1}\right) \in \mathrm{C}^{(j)} .
$$

Gegeben sei ferner eine LPT $\mathfrak{\Omega}_{j}(t)$. Wegen der Voraussetzung (1.3) kann man $\mathfrak{L}_{j}(t)$ auf jede einzelne Realisierung $x(\alpha, t)$ des Prozesses $x(t)$ anwenden und erhält einen neuen Prozeß $y(t)=\{y(\alpha, t)\}$ mit

$$
y(\alpha, t)=\Omega_{j}(t) x(\alpha, t) .
$$

Wir setzen im folgenden stets voraus, daß der Operator $\mathfrak{L}_{j}(t)$ mit dem Operator der statistischen Mittelbildung vertauschbar ist. Dann folgen aus (1.6) mit $(1.4 \mathrm{a}, \mathrm{b})$ folgende Beziehungen zwischen

14 S. Bochner, Fouriersche Integrale, Chelsea Publ. Comp. New York 1948. 
den Mittelwerten und Korrelationsfunktionen der Prozesse $x(t)$ und $y(t)$ :

$$
\begin{aligned}
E_{y}(t)= & \mathfrak{L}_{j}(t) E_{x}(t), \\
R_{y}\left(t, t_{1} \ldots t_{n-1}\right)= & \mathfrak{L}_{j}(t) \mathfrak{L}_{j}^{*}\left(t_{1}\right) \ldots \\
& \mathfrak{S}_{j}\left(t_{n-1}\right) R_{x}\left(t, t_{1} \ldots t_{n-1}\right), \\
R_{x y}\left(t, t_{1}\right)= & \mathfrak{\Omega}_{j}^{*}\left(t_{1}\right) R_{x}\left(t, t_{1}\right) .
\end{aligned}
$$

Dabei bedeutet $\Omega_{j}^{*}(t)$ den zu $\Omega_{j}(t)$ konjugiert komplexen Operator. Man sagt im Hinblick auf (1.6): Die Prozesse $x(t), y(t)$ bilden ein strenges LPPSP.

b) Definition der linear passiven Transformation eines stochastischen Prozesses im statistischen Mittel.

Es gibt physikalische Prozesse, welche sich durch eine linear passive Transformation eines stochastischen Prozesses $x(t)$ beschreiben lassen, welcher die Bedingung (1.3) nicht erfüllt. Ein Beispiel für ein solches System ist ein elektrisches Netzwerk, das eine Elektronenröhre enthält, die statistisch verteilte $\delta$-förmige Stromimpulse abgibt. Strom und Spannung im Netzwerk bilden ein LPPSP15. Deswegen erscheint es zweckmäßig, den Begriff der LPT eines stochastischen Prozesses im statistischen Mittel einzuführen.

Gegeben ist ein stochastischer Proze $\beta x(t)$, definiert über einem Wahrscheinlichkeitsraum

$$
\mathfrak{E}=\{\Omega(\zeta), F(\alpha, \beta \ldots), P(\alpha)\},
$$

dessen Mittelwert und Korrelationsfunktionen beliebiger Ordnung (1.5) genügen, dessen Realisierungen $x(\alpha, t)$ aber nicht (1.3) zu genügen brauchen. Gegeben sei weiterhin eine LPT $\Omega_{j}(t)$. Sie erzeugt gemäß $(1.7 \mathrm{a}, \mathrm{b}, \mathrm{c})$ durch Anwendung auf den Mittelwert und die Korrelationsfunktionen des Prozesses $x(t)$ einen anderen stochastischen Prozeß $y(t)$.

Wir setzen im folgenden stets voraus, daß der Prozeß $y(t)$ bereits durch seinen Mittelwert und sämtliche Korrelationsfunktionen eindeutig bestimmt ist. Diese Beschränkung ist notwendig, damit die Zuordnung $x(t) \rightarrow y(t)$ eindeutig bleibt. Man sagt im Hinblick auf $(1.7 \mathrm{a}, \mathrm{b}, \mathrm{c})$ : Die Prozesse $x(t)$, $y(t)$ bilden ein gewöhnliches LPPSP.

Mit Hilfe zweier Sätze von Pugachev (s. 15a, 15b), welche im wesentlichen eine Anwendung der Ungleichung von BIENAYMÉ $\left({ }^{15}\right.$, p. 151) sind, läßt sich

15 A. Papoulis, Probability Rondom Variables and Stochastic Processes, McGraw-Hill, New York 1965, p. 560.

$15 \mathrm{a}$ vgl. 9 , p. $215 \mathrm{ff}$. zeigen: Bilden die beiden stochastischen Prozesse $x(t), y(t)$ ein gewöhnliches LPPSP, so gibt es eine Folge von algebraischen Operatoren $\Omega j^{(n)}(t)$ mit

$$
\lim _{n \rightarrow \infty} \mathcal{Q}_{j}^{(n)}(t)=\mathfrak{\Omega}_{j}(t)
$$

so daß für beliebig kleine $\varepsilon>0$ und alle $t$

$$
\lim _{n \rightarrow \infty} P\left\{\left|\mathbb{R}_{j}^{(n)}(t) x(t)-y(t)\right|>\varepsilon\right\}=0
$$

ist. Das heißt zu jeder Realisierung $x(\alpha, t)$ des Prozesses $x(t)$ existiert eine Funktion $y(\alpha, t)$ derart, daß die Beziehung

$$
\lim _{n \rightarrow \infty}\left|\mathcal{L}_{j}^{(n)}(t) x(\alpha, t)-y(\alpha, t)\right|<\varepsilon
$$

für alle $\varepsilon>0$ und alle $t$ gilt, wenn nur $\alpha$ aus einer gewissen Ereignismenge $\Lambda$, für welche $P(\Lambda)=1$ gilt, stammt.

Die Funktionen $y(\alpha, t)$ werden als die möglichen Realisierungen des Prozesses $y(t)$ angesehen.

Ausgehend von (1.8) kann leicht gezeigt werden, daß die durch (1.7a, b, c) erzeugte Transformation zwischen den Realisierungen $x(\alpha, t) \rightarrow y(\alpha, t)$ der Prozesse $x(t)$ und $y(t)$ mit Wahrscheinlichkeit 1 linear, kausal und invariant gegenüber Zeittranslation ist. Die Passivitätseigenschaft läßt sich nicht ohne weiteres nachweisen, da die Funktionen $x(\alpha, t)$, $y(\alpha, t)$ bzw. ihr Produkt ja gar nicht integrierbar zu sein brauchen. Stets gelten aber für gewöhnliche LPPSP eine Passivitätseigenschaft der Mittelwerte

$$
\operatorname{Re} \int_{-\infty}^{\tau} E^{*}(t) E_{y}(t) \mathrm{d} t \geqq 0 \ldots \text { alle } \tau
$$

bzw. eine mittlere Passivität

$$
\operatorname{Re} \int_{-\infty}^{\tau} R_{x y}(t, t) \mathrm{d} t \geqq 0 \ldots \text { alle } \tau .
$$

(1.9) folgt aus (1.7a), (1.10) aus (1.7 c) mit Hilfe von (1.2). Die oben gegebene Definition eines gewöhnlichen LPPSP läßt sich noch auf den Fall erweitern, daß die Größen $E_{x}(t)$ und $R_{x}\left(t, t^{\prime}\right)$ verallgemeinerte Funktionen sind. (Beispiel: Thermisches Rauschen $[15$, p. 360$]$.) In diesem Fall muß die charakteristische Funktion $P(s)$ des Operators $\Omega_{j}(t)$ noch weiteren von Fall zu Fall verschiedenen Bedingungen genügen, durch die garantiert wird, daß die das LPPSP definierenden Beziehungen (1.7a, b, c) sinnvoll bleiben ${ }^{16}$.

15b Siehe auch M. LoÉve: Probability Theory D. van Nostrand Comp. N. Y. 1963, Chap. X

16 A. H. Zemanian, Distribution Theory and Transform Analysis, MacGraw-Hill, New York 1965. 


\section{Aussagen über die Statistik des Prozesses $y(t)$}

Gegeben sei ein LPPSP, welches streng oder gewöhnlich, komplex oder reell sein kann. Wir fragen: Welche Aussagen lassen sich über die Statistik des Prozesses $y(t)$ und speziell über Mittelwert und Korrelationsfunktion machen, wenn der Mittelwert und die Korrelationsfunktionen von $x(t)$ und der Operator $\Omega_{j}(t)$ etwa durch Angabe von $a, b$ und $P(s)$ vorgegeben sind?

Wir verwenden im folgenden die Abkürzungen:

A Aussage, B Beweis, V Voraussetzung.

Die Anwendung der Theorie der LPT ${ }^{3,4}$ liefert folgende Aussagen:

A) Darstellung des Mittelwertes $E_{y}(t)$ und der Korrelationsfunktion $R_{y}\left(t, t^{\prime}\right)$ :

Explizite Darstellungen von $E_{y}(t)$ und $R_{y}\left(t, t^{\prime}\right)$ als Funktionale von $E_{x}(t)$ und $R_{x}\left(t, t^{\prime}\right)$ folgen un- mittelbar aus (1.7a, b, c) mit Hilfe von (1.2a, b, c). Wir beschränken uns hier auf einen für die Anwendungen wichtigen Fall einer reellen LPT eines reellen Prozesses und geben $E_{y}(t), R_{y}\left(t, t^{\prime}\right)$ sowie die Streuung $\sigma^{2}(t)$ nur für diesen Fall explizit an:

Abkürzungen:

$$
\begin{gathered}
R_{x}^{0}\left(t, t^{\prime}\right)=R_{x}\left(t, t^{\prime}\right)-E_{x}(t) E_{x}\left(t^{\prime}\right), \\
\dot{P}(k)(s)=\frac{\mathrm{d}^{k}}{\mathrm{~d} s^{k}} P(s), \quad R_{x}^{\prime}\left(t, t^{\prime}\right)=\frac{\partial}{\partial t^{\prime}} R_{x}\left(t, t^{\prime}\right), \\
{ }^{\prime} R_{x}\left(t, t^{\prime}\right)=\frac{\partial}{\partial t} R_{x}\left(t, t^{\prime}\right) .
\end{gathered}
$$

$\mathrm{V}_{1}: E_{x}(t) \in \mathrm{C}^{(2)} \ldots$ reell, $\quad E_{x}(t)=0$ für $t \leqq 0$.

$\mathrm{V}_{2}: \quad R_{x}\left(t, t^{\prime}\right) \in \mathrm{C}^{(2)} \ldots$ reell,$\quad R_{x}\left(t, t^{\prime}\right)=0$ wenn $t \leqq 0$ oder $t^{\prime} \leqq 0$ ist.

$\mathrm{V}_{3}: \mathfrak{L}_{2}(t) \ldots$ reell d.h. $\operatorname{Im} y(1)=0$, $P(s)=P(-s) \ldots$ reell.

Aus (1.2c) folgt mit $(1.7 \mathrm{a}, \mathrm{b})$ :

$$
\begin{aligned}
\mathrm{A}_{1}: \quad E_{y}(t)= & a E_{x}^{\prime}(t)+\int_{0}^{t}[b+P(t-s)] E_{x}(s) \mathrm{d} s+\int_{0}^{t}[P(0)-P(t-s)] E_{x}^{\prime \prime}(s) \mathrm{d} s, \\
\mathrm{~A}_{2}: \quad \sigma_{y}^{2}(t)= & a^{2} R_{x}^{0^{\prime}}(t, t)+2 a b \int_{0}^{t} \mathrm{~d} s^{\prime} R_{x}^{0}(t, t-s)+b^{2} \int_{0}^{t} \int_{0}^{t} R_{x}^{0}\left(t-s_{1}, t-s_{2}\right) \mathrm{d} s_{1} \mathrm{~d} s_{2} \\
& +2 a \int_{0}^{t} \mathrm{~d} s\left[{ }^{\prime} R_{x}^{0}(t, t-s) P(s)+R_{x}^{0^{\prime \prime}}(t, t-s)(P(0)-P(s))\right] \\
& +2 b \int_{0}^{t} \int_{0}^{t} \mathrm{~d} s_{1} \mathrm{~d} s_{2}\left[R_{x}^{0}\left(t-s_{1}, t-s_{2}\right) P\left(s_{1}\right)+{ }^{\prime \prime} R_{x}^{0}\left(t-s_{1}, t-s_{2}\right)\left(P(0)-P\left(s_{1}\right)\right)\right] \\
& +\int_{0}^{t} \int_{0}^{t} \mathrm{~d} s_{1} \mathrm{~d} s_{2}\left\{R_{x}^{0}\left(t-s_{1}, t-s_{2}\right) P\left(s_{1}\right) P\left(s_{2}\right)+2^{\prime \prime} R_{x}^{0}\left(t-s_{1}, t-s_{2}\right)\left(P(0)-P\left(s_{1}\right)\right) P\left(s_{2}\right)\right. \\
& \left.+{ }^{\prime \prime} R_{x}^{0 \prime \prime}\left(t-s_{1}, t-s_{2}\right)\left(P(0)-P\left(s_{1}\right)\right)\left(P(0)-P\left(s_{2}\right)\right)\right\}
\end{aligned}
$$

$\mathrm{A}_{3}: \quad R_{y}\left(t_{1}, t_{2}\right)=a^{2 \prime} R_{x}^{\prime}\left(t_{1}, t_{2}\right)_{4}+a b \int_{0}^{t_{1}} R_{x}^{\prime}\left(t_{1}-s, t_{2}\right) \mathrm{d} s$

$$
\begin{aligned}
& +a b \int_{0}^{t_{2}} \mathrm{~d} s^{\prime} R_{x}\left(t_{1}, t_{2}-s\right)+b^{2} \int_{0}^{t_{1}} \int_{0}^{t_{2}} \mathrm{~d} s_{1} \mathrm{~d} s_{2} R_{x}\left(t_{1}-s_{1}, t_{2}-s_{2}\right) \\
& +a\left\{\int_{0}^{t_{1}} R_{x}^{\prime}\left(t_{1}-s, t_{2}\right) P(s) \mathrm{d} s+\int_{0}^{t_{2}} R_{x}^{\prime \prime}\left(t_{1}, t_{2}-s\right)(P(0)-P(s)) \mathrm{d} s\right. \\
& \left.+\int_{0}^{t_{2}} R_{x}\left(t_{1}, t_{2}-s\right) P(s) \mathrm{d} s+\int_{0}^{t_{1}} R_{x}^{\prime}\left(t_{1}-s, t_{2}\right)(P(0)-P(s)) \mathrm{d} s\right\} \\
& +b \int_{0}^{t_{1} t_{2}} \mathrm{~d} s_{1} \mathrm{~d} s_{2}\left\{R_{x}\left(t_{1}-s_{1}, t_{2}-s_{2}\right)\left(P\left(s_{1}\right)+P\left(s_{2}\right)\right)\right. \\
& \left.+R_{x}^{\prime \prime}\left(t_{1}-s_{1}, t_{2}-s_{2}\right)\left(P(0)-P\left(s_{2}\right)\right)+{ }^{\prime \prime} R_{x}\left(t_{1}-s_{1}, t_{2}-s_{2}\right)\left(P(0)-P\left(s_{1}\right)\right)\right\} \\
& +\int_{0}^{t_{1} t_{2}} \mathrm{~d} s_{1} \mathrm{~d} s_{2}\left\{R_{x}\left(t_{1}-s_{1}, t_{2}-s_{2}\right) P\left(s_{1}\right) P\left(s_{2}\right)+R_{x}^{\prime \prime}\left(t_{1}-s_{1}, t_{2}-s_{2}\right)\left(P(0)-P\left(s_{2}\right)\right) P\left(s_{1}\right)\right. \\
& +{ }^{\prime \prime} R_{x}\left(t_{1}-s_{1}, t_{2}-s_{2}\right)\left(P(0)-P\left(s_{1}\right)\right) P\left(s_{2}\right)+{ }^{\prime \prime} R_{x}^{\prime \prime}\left(t_{1}-s_{1}, t_{2}-s_{2}\right)(P(0) \\
& \left.\left.-P\left(s_{1}\right)\right)\left(P(0)-P\left(s_{2}\right)\right)\right\} .
\end{aligned}
$$


Analoge Darstellungen für $E_{y}(t), R_{y}\left(t_{1}, t_{2}\right)$ und $\sigma^{2}(t)$ lassen sich für den Fall $j=0$ bzw. 1 aus $(1.7 \mathrm{a}, \mathrm{b})$ mit $(1.2 \mathrm{a}, \mathrm{b})$ ableiten.

B) Grenzwertsätze für den Mittelwert $E_{y}(t)^{17}$ :

1. $\mathrm{V}_{1}: E_{x}(t) \in \mathrm{C}^{(j)}, \quad E_{x}(t)=0$ für $t \geqq t_{0}$,

$$
j=0,1,2 .
$$

$\mathrm{V}_{2}: \lim \dot{P}^{(k)}(s)=0$ für $k=0,1, \ldots 2-j$.

$$
\text { A: } \begin{aligned}
\lim _{t \rightarrow \infty} E_{y}(t)= & b \int_{-\infty}^{t_{0}} E_{x}(t) \mathrm{d} t \\
& \text { existiert für alle } j .
\end{aligned}
$$

B: Für $j=2$ siehe ${ }^{3}$, für $j=0,1$ läßt sich der Beweis unter Benutzung von $(1.2 \mathrm{a}, \mathrm{b})$ ganz analog führen.

2. $\mathrm{V}_{1}: E_{x}(t) \in \mathrm{C}^{(j)}, \quad E_{x}(t)=V_{x} e^{i \omega t} \ldots$ für $t \geqq t_{0}$, $V_{x}=$ const, $\omega \ldots$ reell .

$\mathrm{V}_{2}: \lim _{s \rightarrow \infty} \dot{P}^{(k)}(s)=0, \quad k=0,1 \ldots 2-j$.

$\mathrm{V}_{\mathbf{3}}: \quad Y(i \omega)$ existiert (siehe Anhang A).

$$
\text { A: } \begin{aligned}
E_{y}(t) & =G(t)+V_{y} e^{i \omega t} \ldots t \geqq t_{0} \text { mit } \quad V_{y} \\
& =\text { const und } \lim _{t \rightarrow \infty} G(t) \\
& =G(\infty)<\infty .
\end{aligned}
$$

B: Für $j=2$ siehe $^{3}$, für $j=0,1$ läßt sich der Beweis unter Verwendung von $(1.2 \mathrm{a}, \mathrm{b})$ ganz analog führen.

3. $\mathrm{V}_{1}: E_{x}(t) \in \mathrm{C}^{(j)}$.

$\mathrm{V}_{2}: \int_{-\infty}^{\infty}\left|\dot{E}^{(k)}(t)\right| \mathrm{d} t<\infty \ldots k=0,1 \ldots j$.

$\mathrm{V}_{3}: \lim _{s \rightarrow \infty} \dot{P}^{(l)}(s)=0 \quad \ldots l=0,1 \ldots 2-j$.

A: $\lim _{t \rightarrow \infty} E_{y}(t)=b \int_{-\infty}^{\infty} E_{x}(t) \mathrm{d} t$.

B: Für $j=2$ siehe ${ }^{3}$, für $j=0,1$ läßt sich der Beweis ganz analog führen.

4. $\mathrm{V}_{1}: E_{x}(t) \in \mathrm{C}^{(j)}, \quad E_{x}(t)=C_{x} t^{n}+O(t)$ für

$t \geqq t_{0}, C_{x}=$ const, $n>0, \lim _{t \rightarrow \infty} O(t)=0$.

$\mathrm{V}_{2}: \Phi_{2}(\varrho)=0$ (siehe Anhang A).

17 Die unter Punkt B bzw. C angegebenen Sätze sind Anwendungen bzw. Verallgemeinerungen eines Grenzwertsatzes von $\mathrm{KöNIG}^{3}$.

18 G. M. Fichtenholz, Differential- und Integralrechnung, Bd. II, VEB Deutscher Verlag der Wissenschaften, Berlin 1964, p. 585.
$\mathrm{A}:\left|E_{y}(t)\right|<D_{y}+C_{y} t^{n+1} \ldots$ für $t \geqq t_{0}$, $C_{y}, D_{y}=$ const, $j=0,1,2$.

B: Man verwende (1.2), (A.4) und einige einfache Eigenschaften positiv definiter Funktionen $\left({ }^{14}\right.$, p. 74$)$.

5. $\mathrm{V}_{1}: E_{x}(t) \in \mathrm{C}^{(j)}, \quad E_{x}(t)=C_{x}=$ const für $t \geqq t_{0}, \quad j=0,1,2$.

$\mathrm{V}_{2}: \lim _{s \rightarrow \infty} \dot{P}^{(k)}(s)=0, \quad k=0,1 \ldots 2-j$.

$\mathrm{V}_{3}: \Phi_{2}(\varrho)=0, \quad b=0, \quad \Phi_{3}^{\prime}(\varrho)<\infty$ (siehe Anhang A).

A: $\lim _{t \rightarrow \infty} E_{y}(t)=E_{y}\left(t_{0}\right)+C_{x} \int_{0}^{\infty} P(s) \mathrm{d} s$ existiert für alle $j$.

B: Nach (1.2) und (1.7 a) genügt es, die Existenz von $\int_{0}^{\infty} P(s) \mathrm{d} s$ zu zeigen. Dies gelingt mit (V.2) und (V.3) unter Verwendung eines Existenzkriteriums von ABEL ${ }^{18}$ und des Satzes von Riemann-Lebesgue über FourierIntegrale ${ }^{19}$.

C) Grenzwertsätze für die Streuung:

Bemerkung: Bilden die Prozesse $x(t) \rightarrow y(t)$ ein LPPSP, so bilden die Prozesse

$$
x(t)-E_{x}(t) \rightarrow y(t)-E_{y}(t)
$$

auch ein LPPSP.

1. $\mathrm{V}_{1}: R_{x}^{0}\left(t, t^{\prime}\right) \in \mathrm{C}^{(j)}, \quad R_{x}^{0}\left(t, t^{\prime}\right)=0$ wenn $t \geqq t_{0}$ oder $t^{\prime} \geqq t_{0}$.

$\mathrm{V}_{2}: \lim _{t \rightarrow \infty} \dot{P}^{(k)}(t)=0, \quad k=0,1 \ldots 2-j$.

A: $\quad \lim _{t \rightarrow \infty} \sigma_{y}^{2}(t)=b \int_{-\infty}^{t_{0}} \int_{-\infty}^{t_{0}} R_{x}^{0}\left(s_{1}, s_{2}\right) \mathrm{d} s_{1} \mathrm{~d} s_{2}$ existiert für alle $j=0,1,2$.

B: Nach Verwendung von (1.2) und (1.7b) im Falle $j=2$ siehe auch (2.3) - ganz analog zu dem von Satz (B.1) in ${ }^{3}$ gegebenen Beweis durchführbar.

19 S. Bochner u. K. Chandrasekharan, Fourier Transforms (Annals of Mathematics Studies Nr. 19), Princeton University Press 1949, p. 3. 
2. $\mathrm{V}_{1}: R_{x}^{0}\left(t_{1}, t_{2}\right) \in \mathrm{C}^{(j)}$.

$$
\begin{aligned}
\mathrm{V}_{2} & : \int_{-\infty}^{\infty}\left|{ }^{(i)} R_{x}^{0(k)}\left(t, t^{\prime}\right)\right| \mathrm{d} t<\infty, \\
& \int_{-\infty}^{\infty} \int_{-\infty}^{\infty}\left|{ }^{(i)} R_{x}^{0}(k)\left(t, t^{\prime}\right)\right| \mathrm{d} t \mathrm{~d} t^{\prime}<\infty
\end{aligned}
$$

$\mathrm{V}_{2}: \mathrm{V}_{3}$ von Satz Cl.

$$
\text { für alle } i, k=0,1, \ldots j \text {. }
$$

Alle Integrale müssen gleichmäßig bezüglich $t^{\prime}$ konvergieren.

A: $\lim _{t \rightarrow \infty} \sigma_{y}^{2}(t)=b^{2} \int_{-\infty}^{\infty} \int_{\infty}^{0} R_{x}^{0}\left(t, t^{\prime}\right) \mathrm{d} t^{\prime} \mathrm{d} t$

B: Nach Verwendung von (2.3) ganz analog zu dem von Satz (B.3) in ${ }^{3}$ gegebenen Beweis durchführbar.

3. $\mathrm{V}_{1}: R_{x}^{0}\left(t, t^{\prime}\right) \in \mathrm{C}^{(j)}, \quad R_{x}^{0}\left(t, t^{\prime}\right)=r_{x} \geqq 0$

.. Const. wenn $t \geqq t_{0}$ und $t^{\prime} \geqq t_{0}$ ist.

$\mathrm{V}_{2}: \lim _{s \rightarrow \infty} \dot{P}^{(k)}(s)=0, \quad k=0,1 \ldots 2-j$.

$\mathrm{V}_{3}: \Phi_{2}(\varrho)=0, \quad \Phi_{3}^{\prime}(\varrho)<\infty, \quad b=0$

(siehe Anhang A).

$\mathrm{A}: \lim _{t \rightarrow \infty} \sigma_{y}^{2}(t)=\sigma_{y}^{2}\left(t_{0}\right)+r_{x}\left(\int_{0}^{\infty} P(s) \mathrm{d} s\right)^{2}$

$$
\text { ... existiert für alle } j \text {. }
$$

B: Nach Verwendung von (1.2) und (1.7 b) analog durchführbar wie der Beweis von Satz B.5.

D) Darstellung der im statistischen Mittel investierten bzw. verlorenen Energie:

1. Der Realteil des Zeitintegrals der gemischten Korrelationsfunktion, genommen über das Intervall $(-\infty, \tau)$, stellt bei vielen Anwendungen die im statistischen Mittel innerhalb dieses Zeitraumes von außen in das System investierte Energie dar. Für dieses Integral gilt folgende Darstellung:

$$
\begin{aligned}
\mathrm{A}_{1}: & \operatorname{Re} \int_{-\infty}^{\tau} R_{x y}(t, t) \mathrm{d} t=\frac{1}{2} a R_{x}(\tau, \tau) \\
& +\frac{1}{2} b \int_{-\infty}^{\tau} \int_{-\infty}^{\tau} R_{x}\left(t, t^{\prime}\right) \mathrm{d} t \mathrm{~d} t^{\prime} \\
+ & \frac{1}{2} \int_{-\infty}^{\infty}\left(1+\varrho^{2}\right) \int_{-\infty}^{\tau} \int_{-\infty}^{\tau} \mathrm{e}^{i \varrho\left(t-t^{\prime}\right)^{\prime}} R_{x}\left(t, t^{\prime}\right) \mathrm{d} t \mathrm{~d} t^{\prime} \mathrm{d} \Phi(\varrho),
\end{aligned}
$$

$\Phi(\varrho)$ ist die Spektralfunktion der LPT (siehe Anhang A).

Die Darstellung (2.13) gilt für $j=1,2$. Im Falle $j=0$ ist sie gültig, wenn $a=0$ gesetzt wird.
B: Man erhält (2.13) unmittelbar durch Anwendung von (1.2) auf (1.7 c).

$$
\begin{gathered}
\mathrm{A}_{2}: \quad \operatorname{Re} \int_{-\infty}^{\tau} R_{x y}(t, t) \mathrm{d} t \geqq \operatorname{Re} \int_{-\infty}^{\tau} E_{x}^{*}(t) E_{y}(t) \mathrm{d} t \geqq 0 \\
\ldots \text { alle } \tau .
\end{gathered}
$$

B: Dies folgt aus (1.10), da auch

$$
x(t)-E_{x}(t) \rightarrow y(t)-E_{y}(t)
$$

ein LPPSP bilden. Die im statistischen Mittel investierte Energie ist stets größer oder gleich der beim Anlegen des statistischen Mittelwertes investierte Energie.

2. Wie nennen ein LPPSP irreversibel, wenn die das Paar erzeugende LPT $\Omega_{j}(t)$ irreversibel im Sinne von KöNIG und ToBergte 11 ist. Bei physikalischen Systemen, welche durch ein irreversibles LPPSP beschrieben werden (Beispiel: Brownsche Bewegung), ist die von außen in das System investierte Energie nicht mehr vollständig zurückgewinnbar. Die im statistischen Mittel im Zeitraum $(-\infty, \tau)$ verlorene Energie ${ }^{11}$ ist gegeben durch

$$
\begin{gathered}
E\{V(x(\zeta, t),-\infty<t<\tau)\}=\pi \int_{-\infty}^{\tau} \mathrm{d} t \int_{0}^{t} \int_{0}^{t} \mathrm{~d} s_{1} \mathrm{~d} s_{2} \\
\cdot h^{*}\left(t-s_{1}\right) h\left(t-s_{2}\right) \\
\cdot\left\{R_{x}\left(s_{1}, s_{2}\right)+R_{x}^{\prime}\left(s_{1}, s_{2}\right)+{ }^{\prime} R_{x}\left(s_{1}, s_{2}\right)\right. \\
\left.+R_{x}^{\prime}\left(s_{1}, s_{2}\right)\right\} \geqq 0 .
\end{gathered}
$$

Die beim Anlegen des statistischen Mittelwertes verlorene Energie ist gegeben durch

$$
\begin{gathered}
V\left(E_{x}(t),-\infty<t \leqq \tau\right)=\pi \int_{-\infty}^{\tau} \mathrm{d} t \int_{0}^{t} \int_{0}^{t} \mathrm{~d} s_{1} \mathrm{~d} s_{2} \\
\cdot h^{*}\left(t-s_{1}\right) h\left(t-s_{2}\right) \\
\cdot\left\{E_{x}\left(s_{1}\right) E_{x}^{*}\left(s_{2}\right)+E_{x}^{\prime}\left(s_{1}\right) E_{x}^{*}\left(s_{2}\right)\right. \\
\left.+E_{x}\left(s_{1}\right) E_{x}^{*}\left(s_{2}\right)+E_{x}^{\prime}\left(s_{1}\right) E_{x}^{* \prime}\left(s_{2}\right)\right\} \geqq 0 .
\end{gathered}
$$

Dabei bedeutet $h(t-s)$ die ,,äußere Funktion“ des Operators $\Omega_{j}(t)^{11}$. Analog zu (2.14) läßt sich folgende Beziehung beweisen:

$$
\begin{aligned}
E\{V(x(\zeta, t), & -\infty<t \leqq \tau)\} \\
& \geqq V\left(E_{x}(t),-\infty<t \leqq \tau\right) \geqq 0 .
\end{aligned}
$$

B : Man ersetze in (2.15) $x(\zeta, t) \rightarrow x(\zeta, t)-E_{x}(t)$ bzw. $R_{x} \rightarrow R_{x}^{0}$ und beachte (2.16).

E) Schranken für die einfache Verteilungsfunktion $F_{y}\left(y_{0}, t\right)$ des Prozesses $y(t)$ :

Nach $(1.7 \mathrm{a}, \mathrm{b})$ und (1.2) lassen sich $E_{y}(t)$ und $\sigma_{y}^{2}(t)$ aus $E_{x}(t)$ und $R_{x}\left(t_{1}, t_{2}\right)$ berechnen. Aus der Kenntnis von $E_{y}(t)$ und $\sigma_{y}^{2}(t)$ folgen Schranken für die Verteilungsfunktion $F_{y}\left(y_{0}, t\right)$, welche die Wahr- 
scheinlichkeit dafür angibt, daß zur Zeit $t y(t)$ im Intervall $\left(-\infty, y_{0}\right)$ liegt:

$F_{y}\left(y_{0}, t\right) \leqq \frac{\sigma_{\nu}^{2}(t)}{\sigma_{y}^{2}(t)+\left(y_{0}-E_{y}(t)\right)^{2}} \ldots E_{y}(t)>y_{0}$,

$F_{y}\left(y_{0}, t\right) \geqq \frac{\left(y_{0}-E_{y}(t)\right)^{2}}{\sigma_{y}^{2}(t)+\left(y_{0}-E_{y}(t)\right)^{2}} \ldots E_{y}(t)<y_{0}$.

Diese Schranken lassen sich analog zu den in $\mathbf{1 5}$, p. 162 angegebenen leicht begründen.

F) Diskussion der mittleren Korrelationsfunktion $\varrho_{x y}(\tau)$ bzw. $\varrho_{y}(\tau)$ :

1. $\mathrm{V}: \int_{-\infty}^{\infty} R_{x}^{1 / 2}(t, t) \mathrm{d} t<\infty, \quad R_{x}^{1 / 2}(t, t) \geqq 0 ;$

Definition: $\varrho_{x}(\tau)=\int R_{x}(t, t+\tau) \mathrm{d} t$.

$\mathrm{A}_{1}: \varrho_{x y}(\tau)=\int_{-\infty}^{\infty} R_{x y}(t, t+\tau) \mathrm{d} t=\varrho_{y x}^{*}(-\tau)$

... existiert für alle $\tau$.

$\mathrm{A}_{2}: \varrho_{x y}(\tau)=\Omega_{j}^{*}(\tau) \varrho_{x}(\tau), \quad j=0,1,2$.

B: Man verwende (1.7c) mit (1.2), einige einfache Sätze über die Vertauschbarkeit von Differential und Integraloperatoren 18 und die folgenden Ungleichungen:

$$
\begin{aligned}
\left|R_{x}^{(i)}\left(t_{1}, t_{2}\right)\right|^{2} \leqq & R_{x}\left(t_{1}, t_{1}\right)^{(i)} R_{x}^{(i)}\left(t_{2}, t_{2}\right), \\
& i=0,1,2 . \\
\left|R_{x y}\left(t_{1}, t_{2}\right)\right|^{2} \leqq & R_{x}\left(t_{1}, t_{1}\right) R_{y}\left(t_{2}, t_{2}\right), \\
& |P(s)| \leqq P(0) .
\end{aligned}
$$

2. V: $\int_{-\infty}^{\infty}\left[{ }^{(i)} R_{x}^{(i)}(t, t)\right]^{1 / 2} \mathrm{~d} t<\infty \quad$ für $\quad i=0,1,2$.

Alle Wurzeln sind stets positiv zu nehmen.

$$
\begin{gathered}
\mathrm{A}_{1}: \varrho_{y}(\tau)=\int_{-\infty}^{\infty} R_{y}(t, t+\tau) \mathrm{d} t=\varrho_{y}^{*}(-\tau) \\
\ldots \text { existiert für alle } \tau .
\end{gathered}
$$

$\mathrm{A}_{2}: \varrho_{y}(\tau)=\mathfrak{\Omega}_{j}^{*}(\tau) \varrho_{x y}(\tau)=\mathfrak{\Omega}_{j}^{*}(\tau) \Omega_{j}(-\tau) \varrho_{x}(\tau)$ alle $j=0,1,2$.

B: Man verwende (1.7 b, c) mit (1.2), Hilfssätze über die Vertauschbarkeit von Differential und Integraloperatoren und die bei Satz F.1 angegebenen Ungleichungen.

$\mathrm{A}_{3}: \quad \operatorname{Re} \varrho_{x y}(0) \geqq 0$.

$\mathrm{A}_{4}: \quad\left[\operatorname{Re} \varrho_{x y}(\tau)\right]^{2} \leqq \varrho_{x}(0) \varrho_{y}(0)$.

$\mathrm{A}_{5}: \quad \operatorname{Re}\left(\varrho_{x y}(\tau)+\varrho_{x y}(-\tau)\right) \leqq 2 \operatorname{Re} \varrho_{x y}(0)$.

B: $A_{3}$ folgt aus (1.10), $A_{4}$ aus der Definition von $\varrho_{x}(\tau), \varrho_{x y}(\tau)$ und $\varrho_{y}(\tau), \mathrm{A}_{5}$ folgt aus (1.10), wenn $\operatorname{man} x(t) \rightarrow x(t)-x(t+\tau)$ ersetzt.
Es lassen sich noch weitere Aussagen über die Statistik des Prozesses $y(t)$ machen. So z. B. folgen aus (1.2) Sätze über die Spektralfunktionen, die charakteristischen Funktionen und auch über das charakteristische Funktional des Prozesses $y(t)$. Ferner läßt sich die Theorie der LPT stochastischer Prozesse nicht nur für skalare Prozesse, sondern auch für vektorielle Prozesse formulieren. Auf diese Fragen soll in einer folgenden Arbeit eingegangen werden.

\section{Anhang A}

Jedem linear passiven Operator $\mathfrak{\Omega}_{j}(t) \neq 0$ kann umkehrbar eindeutig eine positive Funktion $Y(p)$ zugeordnet werden ${ }^{3}$. Diese Funktion ist analytisch in $\operatorname{Re} p>0$, und es gilt $\operatorname{Re} Y(p)>0$ in $\operatorname{Re} p>0$. $Y(p)$ besitzt eine eindeutige Darstellung.

$Y(p)=i \operatorname{Im} Y(1)+a p+\frac{b}{p}+\int_{-\infty}^{\infty} \frac{1-i p \varrho}{p-i \varrho} \mathrm{d} \Phi(\varrho)$.

Dies gilt für $j=1,2$. Im Falle $j=0$ ist in (A.1) die Konstante $a=0 \mathrm{zu}$ ersetzen. Es bedeuten dabei $Y(1), a \geqq 0, b \geqq 0$ Konstante und $\Phi(\varrho)$ eine Spektralfunktion ${ }^{3,14}$, welche eindeutig in 3 verschiedene Anteile zerlegt werden kann:

$$
\Phi(\varrho)=\Phi_{1}(\varrho)+\Phi_{2}(\varrho)+\Phi_{3}(\varrho) .
$$

$\Phi_{1}(\varrho)$ ist eine reine Sprungfunktion, $\Phi_{3}(\varrho)$ eine fast überall differenzierbare Funktion. $\Phi_{2}(\varrho)$ ist der ,,pathologische" Anteil, welcher überall stetig aber nirgendwo differenzierbar ist. Dieser Anteil scheint bei physikalischen Anwendungen nur eine geringe Rolle zu spielen. Die charakteristische Funktion ist definiert durch das Fourier-Stiltjes-Integral von $\Phi(\varrho)$

Es gilt ${ }^{3,14}$

$$
P(s)=\int_{-\infty}^{\infty} e^{i s \varrho} \mathrm{d} \Phi(\varrho) .
$$

$$
\begin{gathered}
|P(s)|<P(0)=\Phi(\infty)-\Phi(-\infty)<\infty, \\
\lim _{t \rightarrow \infty} \frac{1}{t} \int_{0}^{t} P(s) \mathrm{d} s=0 .
\end{gathered}
$$

Aus (A.1) und (A.3) folgt eine 2. Darstellung von $Y(p)$

$$
\begin{aligned}
Y(p)= & i \operatorname{Im} Y(1)+a p+\frac{b}{p}+\int_{0}^{\infty} e^{-p t} P(t) \mathrm{d} t \\
& -p^{2} \int_{0}^{\infty}[P(t)-P(0)] e^{-p t} \mathrm{~d} t .
\end{aligned}
$$

Für positiv reelle Funktionen $Y(p)$ ist $\operatorname{Im} Y(1)=0$ und $\Phi(\varrho)=-\Phi(-\varrho)$. 